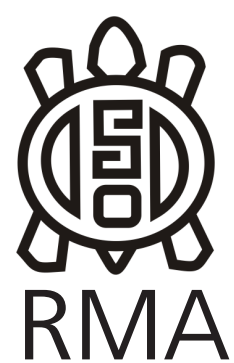

Arqueología

\title{
Materias primas líticas en la Costa Norte del Golfo San Matías (Río Negro, Argentina): distribución de fuentes y tendencias generales en su aprovechamiento
}

\author{
Cristian M. Favier Dubois* y Jimena Alberti**
}

*INCUAPA-CONICET. cfavier3@gmail.com. **IMHICIHU-CONICET. jimealberti@gmail.com

\begin{abstract}
Resumen
La costa norte del golfo San Matías (Río Negro, Argentina) presenta fuentes secundarias de materias primas líticas aptas para la talla, casi ubicuas en el ambiente. Estos conjuntos constituyen rocas redepositadas por acción marina, fluvial o glacifluvial, son de extensión variable y poseen litología diversa. En este trabajo se describen las fuentes a partir de la información de las cartas geológicas, de bibliografía especifica, y de relevamientos propios. Además se analiza la influencia de las variaciones en la línea de costa en la disponibilidad y accesibilidad a las mismas, y se incluye la identificación petrográfica de las rocas. Finalmente, se delinean tendencias generales en su aprovechamiento. Los resultados indican que fueron las rocas silíceas las más utilizadas, seguidas de las vulcanitas y de las calcedonias en tercer lugar. Asimismo, se registraron diferencias en la explotación en los dos bloques temporales reconocidos para el uso de los recursos costeros en este litoral.
\end{abstract}

Palabras clave: costa norte del golfo San Matías; fuentes de materias primas líticas; explotación de rocas; acreción costera.

Lithic raw materials in the northern coast of San Matias Gulf (Río Negro, Argentina): sources distribution and general trends in their exploitation

\begin{abstract}
The northern coast of San Matías Gulf (Río Negro, Argentina) has secondary sources of lithic raw materials suitable for flake activities, which are almost ubiquitous in the environment. These rocks sets have been redeposited by marine, fluvial or glaciofluvial action. Also, they are of variable length and possess diverse lithology. In this paper we describe the sources using information from geological maps, specific bibliography, and our surveys in the field. Furthermore, we examine the influence of variations in the coast line on the availability and accessibility to these sources, and include different rocks petrographic identification. Finally, we outline general trends in their use. The results indicate that siliceous rocks were the most exploited, followed by vulcanites and chalcedonies in third position. There were also differences in the rock exploitation in the two temporary blocks recognized for the coastal resources use in this coast.
\end{abstract}

Keywords: northern coast of San Matías Gulf; lithic raw material sources; rocks exploitation; coastal accretion.

La costa del golfo San Matías, en la provincia de Río Negro, ha sido objeto de estudios arqueológicos sistemáticos desde el año 2004. Las investigaciones se han enfocado principalmente en el sector norte de la mencionada área, que abarca desde la localidad de San Antonio Oeste hasta Bahía Rosas. Los trabajos en el sector oeste del golfo, desde San Antonio Oeste hasta el límite con la provincia de Chubut, se han intensificado recientemente. A partir de estas investigaciones, se han propuesto para la costa norte dos períodos diferenciados respecto al consumo de los recursos marinos sobre la base, principalmente, de estudios isotópicos (paleodietas humanas). El primero de ellos comprende el lapso que se ubica entre los 4.800 y los 2.200 años ${ }^{14} \mathrm{C}$ AP y habría implicado un uso intensivo de la costa y de los recursos disponibles en ella que se remontaría a unos 6.000 años ${ }^{14} \mathrm{C}$ AP de acuerdo con la evidencia arqueológica disponible (Favier Dubois et al. 2009a; Favier Dubois y Scartascini 2012). Como lo evidencia la aparición conspicua de pesas líticas de manufactura simple a lo largo de la costa del área estudiada, durante ese período se habrían aprovechado principalmente los peces, aunque 
también hay evidencias del consumo de mamíferos marinos, aves marinas, moluscos y crustáceos (Favier Dubois et al. 2009a; Scartascini 2010).

Para el segundo período, que abarca entre los 1.500 y los 450 años ${ }^{14} \mathrm{C} A P$, se registra en los sitios una mayor evidencia de consumo de fauna terrestre (guanaco, rheidos y pequeños mamíferos), que se suma al de fauna marina que se verifica desde el bloque anterior (Favier Dubois et al. 2009a; Marani y Scartascini 2011). La cerámica y el cambio en la morfología y tamaño de las puntas de proyectil son rasgos tecnológicos conspicuos de este período (Favier Dubois et al. 2009a).

Se ha propuesto que estos cambios paleodietarios se vinculan con cambios en la morfología costera, particularmente el relleno de los entrantes marinos durante los pulsos de descenso del nivel del mar en el litoral Atántico de Patagonia (ver más adelante Schellmann y Radtke 2010). Este relleno condujo a una rectificación paulatina de la costa a la que se asocian cambios en las tecnologías de pesca; en particular se registra evidencia de un mayor uso de líneas de pesca en momentos tardíos, en detrimento del uso de redes que era favorecido por la presencia de numerosos entrantes en la costa más irregular existente durante el período temprano (Favier Dubois y Kokot 2011). A esta evidencia tecnológica se suma evidencia ictioarqueológica, ya que el número y características de los otolitos de corvina rubia (Micropogonias furnieri) en uno y otro bloque respaldan tal cambio en las prácticas pesqueras (Favier Dubois y Scartascini 2012).

A lo largo de la duración de la ocupación del área, la disponibilidad y accesibilidad a las fuentes de materias primas líticas ha sufrido cambios debido a la dinámica propia de las áreas costeras. Esto habría influenciado las estrategias de aprovechamiento de las rocas por parte de los grupos que habitaron el área.

El objetivo de este trabajo es presentar una caracterización de las fuentes de materias primas aptas para la talla en la costa norte del golfo San Matías. Para ello se llevó a cabo una revisión bibliográfica exhaustiva de autores que han trabajado en el área y, en base a ello, se realizaron muestreos y observaciones en el campo. Además, se relevaron y analizaron los conjuntos líticos provenientes de localidades arqueológicas ubicadas a lo largo de toda la costa para lograr un acercamiento a la tecnología lítica manufacturada y utilizada por los grupos cazadores-recolectores que habitaron la zona. Finalmente, se realizaron cortes delgados para identificar microscópicamente a las rocas mediante el uso de un microscopio petrográfico. De esta manera, se logró una caracterización precisa de la variabilidad de rocas aptas para la talla recuperadas tanto en las fuentes como en los sitios arqueológicos en sí.

\section{Morfología costera}

La costa norte del golfo San Matías presenta planicies elevadas de rodados, cordones medanosos, bajos y una zona litoral, en la que se alternan playas con fácil acceso al mar con acantilados abruptos, cuyas alturas oscilan entre los 3 y los 30 msnm (González Díaz y Malagnino 1984). La planicie aluvial disectada mencionada es la que ha sido denominada Meseta Patagónica, situada entre los 140 y los 170 msnm con una pendiente suave que se inclina hacia el NO-SO (Angulo et al. 1978; González Díaz y Malagnino 1984). Esta planicie se encuentra coronada por un manto de grava (Rodados Patagónicos) y presenta interrupciones (bajos), generalmente ocupados por agua o por sales (Angulo et al. 1981). Estos bajos, entre los que se destacan el Gran Bajo del Gualicho, el Bajo de Valcheta y el Bajo de San Antonio, poseen dimensiones que van desde los 1-2 km hasta los $10 \mathrm{~km}$ de diámetro, con profundidades que alcanzan un máximo de $30 \mathrm{~m}$ (Angulo et al. 1981; Gelós et al. 1990). Algunos de ellos, como es el caso del Bajo de San Antonio, configuran importantes entrantes costeros (González Díaz y Malagnino 1984).

En lo que hace a la zona litoral propiamente dicha, la misma presenta rasgos en general por debajo de la curva de nivel de los 20 m, divididos en unidades de origen eólico y unidades de origen marino (Angulo et al. 1978; Gelós et al. 1990). Entre las primeras se ubican los médanos (fijos-semifijos y activos) y mantos sobre los cordones litorales y las llanuras de marea antiguas (Angulo et al. 1978, 1981; Gelós et al. 1990). Existen además en el área geoformas de origen marino, que pueden ser divididas en antiguas (cordones litorales y las playas y llanuras de marea) y recientes (playas, acantilados, plataformas de abrasión, espigas, canales, plataforma sub-litoral y llanura de marea) (Angulo et al. 1978, 1981; Gelós et al. 1990). En la zona de playas y paleoplayas donde se desarrollan los cordones litorales se encuentran los principales depósitos secundarios de rocas que habrían funcionado como fuentes potenciales de materia prima lítica apta para la talla (Alberti 2012).

Los cordones litorales correspondientes al retroceso marino posterior al máximo transgresivo del Holoceno conforman las terrazas más bajas (3-10 msnm) (Favier Dubois et al. 2008), mientras que las altas (15-30 msnm) corresponden a cordones y plataformas litorales elevadas de edad pleistocena (Rostami et al. 2000) (Figura 1).

\section{Formaciones geológicas de interés arqueológico presentes en el área de estudio}

A partir de la revisión de la bibliografía pertinente, se ha recopilado información geológica respecto a las unidades aflorantes y, en base a ello, a la disponibilidad de recursos líticos aptos para la talla en la costa norte del golfo San Matías. A continuación se presentan las formaciones geológicas que revisten interés arqueológico, 


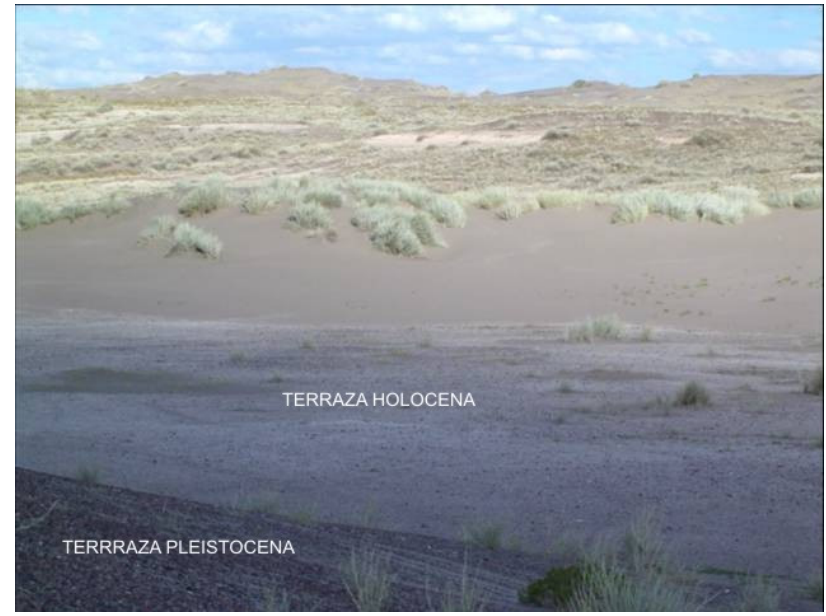

Figura 1. Terrazas holocena y pleistocena en Bajo de la Quinta.

Figure 1. Holocene and Pleistocene terraces in Bajo de la Quinta.

en el orden de sus edades relativas, de las más antiguas a las más recientes. Cabe destacar que en los casos en los que corresponde, se menciona la extensión de la formación hacia la costa oeste del golfo, aunque las unidades geológicas correspondientes a esta área serán desarrolladas en una futura contribución.

\section{Formación Patagonia (sensu lato)}

Está integrada por tobas cristalinas y cristalinovítreas, tobas calcáreas, arcilitas, algunos bancos de coquina, un banco de tufita calcárea muy silicificada, y limolitas amarillo verdosas con intercalaciones arcillosas, de origen marino, correspondientes al Mioceno (Camacho 1967; Feruglio 1950; Weber 1983). Por lo general posee fósiles, siendo frecuente la presencia de Ostrea sp., así como de erizos y gasterópodos. En la base de la secuencia se encuentra un conjunto de tufitas calcáreas muy silicificadas $(0,50 \mathrm{a}$ $1 \mathrm{~m}$ de espesor) que presentan un bandeado con capas alternantes de material silíceo, calcedonia y ópalo, cuyo color general es gris blanquecino, ocráceo y hasta rojizo (Weber 1983).

Fidalgo y Porro (1981) establecieron diferentes facies desde el tope hacia la base: sedimentos arenosos de la Fm. Punta Villarino (eólicos) en discordancia de erosión; sedimentos limoarenosos a limoarcillosos de color verdoso; sedimentos similares a los anteriores pero más compactados y con yeso; sedimentos arenosos de aspecto masivo; sedimentos estratificados arcillosos de color verdoso; y sedimentos limo-arenosos a limo-arcillosos con concreciones de óxido de hierro de color castaño. El techo de esta formación se relaciona en forma transicional con la Fm. Río Negro de carácter continental (Fidalgo y Porro 1981).

La Fm. Patagonia se distribuye en la costa norte del golfo a lo largo de unos 15 km a la altura de Barranca Final, tanto en la plataforma de abrasión como en el acantilado (ver 6 en la Figura 2). En la costa oeste aflora entre Punta Delgado y unos $2 \mathrm{~km}$ al sur de Las Grutas, y luego en forma discontinua hasta las barrancas del arroyo Salado (Fidalgo y Porro 1981; Weber 1983).

En la costa norte del golfo las facies aflorantes correspondientes a la clásica Fm. Patagonia se han denominado Formación Barranca Final, corresponden al Mioceno medio, y se hallan compuesta por arcilitas blanquecinas y oscuras con intercalaciones tobáceas, y algo de fauna (principalmente Scapharca bonplandiana) (Franchi 1983; Kaasschieter 1965; Malumián y Nañez 2011).

\section{Formación Río Negro (Andreis 1965)}

La conforman tobas calcáreas, cristalinas, cineríticas, y conglomerados. Hay además areniscas de grano fino a mediano, gris azuladas a gris pardas, con estratificación laminar a entrecruzada, arcilitas limosas finamente estratificadas de colores gris blanquecino, pardo claro y rosado, pelitas y psefitas (Franchi 1983; Gelós et al. 1992; Weber 1983; Zavala y Freije 2005). Los clastos que conforman las areniscas son de pastas volcánicas (riolitas, andesitas, basaltos, vidrio volcánico). Diferentes autores le han asignado distintas edades: miocena, pliocena o cuartaria. De acuerdo con Franchi (1983) está datada en el Plioceno medio a superior.

Wichman (1918) la ha definido como una arenisca gris azulada compuesta de granos de cuarzo, pórfido oscuro, magnetita y hornblenda, que en contacto con el mar adquiere una coloración verdosa. En partes presenta poca consistencia, mientras que en otras hay bancos compactos debido al carbonato de calcio (Wichman 1918). En este sentido, los sedimentos son friables, pero intercalados en las capas entrecruzadas se observan lajones horizontales muy duros de hasta $20 \mathrm{~cm}$ de espesor, cementados por carbonato de calcio (Sánchez 1973).

La Fm. Río Negro se distribuye sólo en la costa norte del golfo, desde la desembocadura del río Negro hasta Barranca Final (ver 4 en la Figura 2), constituyendo la totalidad o gran parte de las sedimentitas observables en el acantilado marino y plataforma de abrasión. Aflora además en los bordes de las mesetas por debajo de la Fm. Tehuelche y por encima de la Fm. Patagonia. Los bancos cementados de estas areniscas son los de interés arqueológico ya que fueron utilizados para la manufactura de artefactos de molienda (ver Orlando 2009). Sin embargo, no hemos observado en los acantilados de las localidades arqueológicas las partes más consolidadas mencionadas por estos autores. Si se han encontrado lajones de esta materia prima, abundantes y disponibles entre los materiales psefíticos de los cordones litorales holocenos, algunos con tamaños aptos para la confección de molinos o morteros (ver Orlando 2009). 


\section{Formación Tehuelche (Fidalgo y Riggi 1970; Weber 1983)}

Esta formación se encuentra constituida por gravas arenosas con cemento calcáreo que corresponden a una antigua planicie aluvial hoy disectada. Ha sido datada en el Plioceno superior/Pleistoceno (Del Río et al. 2005; Martínez et al. 2001). Dentro de la formación existen dos tipos de depósitos: los que integran la meseta principal y los de los pedimentos de flanco, los cuales son un redepósito de los anteriores (Fidalgo y Porro 1981).

Esta formación es proveedora de rodados gruesos, muchos de los cuales presentan costras carbonáticas. Además se observan con frecuencia texturas superficiales en la forma de marcas semilunares producto del acarreo fluvial. Aunque su origen es discutido, la petrofábrica de estos rodados indica que no responden a gravas depositadas por glaciares sino que la depositación fue en medio ácueo (Cortelezzi et al. 1963). De acuerdo con Franchi (1983), su origen sería fluvial, involucrando la sedimentación y la remoción en masa.

Los clastos, de grano fino en general, son redondeados a sub-redondeados, y están integrados por vulcanitas básicas a ácidas (riolitas, dacitas, andesitas y basaltos), plutonitas, cuarcitas y sedimentitas, predominando los basaltos y pórfidos (Cortelezzi et al. 1963; Fidalgo y Porro 1981; Martínez et al. 2001). Con baja frecuencia se observan los rodados originados en rocas calcedónicas, lava vesiculosa y piedra pómez (Sánchez 1973), rocas filonianas de tipo aplítico y rodados de cuarzo y feldespato potásico (Cortelezzi et al. 1963). Una muestra obtenida por Etcheverría et al. (2006) en el tramo oeste de la costa norte reveló $48 \%$ de basalto, $40 \%$ de andesitas, $10 \%$ de riolitas, $2 \%$ de cuarzo y aislados clastos de areniscas de la Fm. Río Negro, y en un perfil realizado por estos autores al norte del río Negro se menciona la presencia de clastos de ópalo, que se hacen abundantes en el techo de la secuencia (Etcheverría et al. 2006:14). Los clastos más grandes oscilan en promedio entre 3 y $5 \mathrm{~cm}$ de diámetro (Angulo et al. 1978), alcanzando medidas entre los $10 \mathrm{y}$ $14 \mathrm{~cm}$ en su eje mayor (Busteros et al. 1998; Etcheverría et al. 2006).

Respecto de su distribución, en la costa norte rematan los acantilados entre la desembocadura del río Negro (en ambas márgenes) y Barranca Final, cubriendo en discordancia a las Formaciones Río Negro y Patagonia con una potencia máxima de 2,5 m (Franchi 1983; Gelós et al. 1992) (ver 5 en la Figura 2). En general, hacia la costa los depósitos disminuyen su espesor y los rodados son de tamaños menores (Fidalgo y Porro 1981). El arroyo Salado (en la costa oeste del golfo) marca el límite de dispersión para la formación, ya que al sur de su curso no es frecuente encontrarla (Weber 1983). Esta formación ha sido la principal proveedora de guijarros para los grupos humanos que habitaron la costa norte del golfo San Matías -ya sea en forma directa o indirecta (retrabajo marino)- resultando principalmente de interés los guijarros de rocas volcánicas de variadas calidades para la talla (ver infra).

\section{Formación Baliza San Matías (Angulo et al. 1978)}

Conglomerado bien estratificado de matriz arenolimosa, fuertemente cementado por carbonato de calcio, de color castaño oscuro a castaño amarillento, compuesto por rodados y valvas de moluscos enteras o fragmentadas

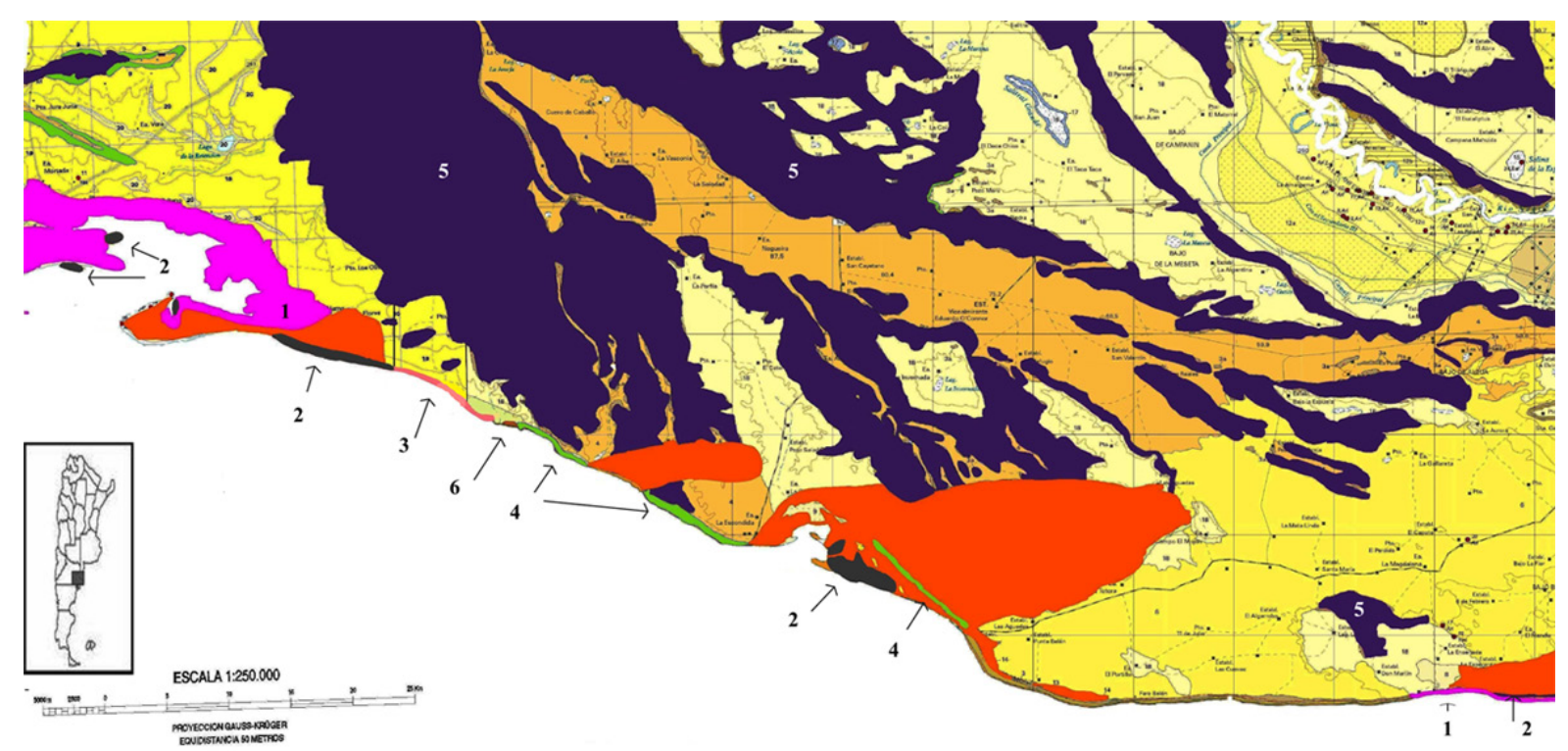

Figura 2. Hoja geológica con las principales formaciones geológicas de la costa norte del golfo San Matías. 1. Fm. San Antonio. 2. Fm. Baliza San Matías mapeada en las hojas. 3. Extensión real de la Fm. Baliza San Matías, mapeada en el campo. 4. Fm. Río Negro. 5. Fm. Manto Tehuelche (Rodados Patagónicos). 6. Fm. Patagonia.

Figure 2. Geological map with the main geological formations in the northern coast of San Matías Gulf. 1. San Antonio fm. 2. Baliza San Matías $\mathrm{fm}$ as it appears in the maps. 3. Real extention of Baliza San Matías fm as mapped in the field. 4. Río Negro fm. 5. Manto Tehuelche fm (Rodados Patagónicos). 6. Patagonia fm. 
(frecuentemente Amiantis purpurata y Mactra sp.) (Angulo et al. 1978; Fidalgo y Porro 1981; Fidalgo y Rabassa 1984). Es un depósito de origen marino y está datado en el Pleistoceno.

Los rodados de esta unidad provienen fundamentalmente de rocas ígneas como basaltos y pórfidos. Sus diámetros oscilan entre pocos milímetros y más de $20 \mathrm{~cm}$, siendo los más comunes de 3 a $5 \mathrm{~cm}$ (Angulo et al. 1978; Martínez et al. 2001). Los clastos son subredondeados y asimétricos, presentando un bajo índice de aplanamiento (Angulo et al. 1978), y provienen del retrabajo marino de rodados de la Fm. Tehuelche (Gelós et al. 1992). El espesor de esta unidad oscila entre 1 y 2 m aunque en Caleta Falsa (Punta Villarino) alcanza casi los 4 m (Angulo et al. 1978; Fidalgo y Rabassa 1984).

Esta formación se apoya sobre la Fm. Patagonia y es cubierta por la Fm. San Antonio (Fidalgo y Porro 1981). Se distribuye siempre en la plataforma de abrasión de ola por lo que se observa particularmente en los momentos de baja marea. En la costa norte aflora desde Saco Viejo hasta Barranca Final (Fidalgo y Porro 1981) (ver Figura 2). Cabe destacar que esta formación se extiende 11 $\mathrm{km}$ más hacia el este de lo que se informa en las hojas geológicas, llegando hasta los 40 $53^{\prime} 59.7^{\prime \prime}$ de latitud sur y los 64 $27^{\prime} 58.3^{\prime \prime}$ de longitud oeste (Alberti 2012) (ver 2 y 3 en la Figura 2). Esta formación ha sido también proveedora de guijarros para la talla, no tanto en forma directa debido a la cementación del conglomerado, sino indirecta mediante su erosión por el oleaje y distribución de los guijarros como rodados costeros y cordones.

\section{Formación San Antonio (Angulo et al. 1978)}

Formación compuesta por grava arenosa, arena y abundantes valvas. Las especies más frecuentes son Glycimeris longior, Chlamys sp., Piter rostrata y Crepidula protea (Angulo et al. 1978). Esta formación conforma playas elevadas y cordones hasta una altitud de 15 a 20 msnm correspondientes al Pleistoceno superior/ Holoceno, cubiertos por sedimentos eólicos de diferente espesor (Fidalgo y Rabassa 1984; Gelós et al. 1992) (ver 1 en la Figura 2). Su altura y las dataciones obtenidas en esta unidad la restringen en la actualidad al Pleistoceno superior (Favier Dubois y Kokot 2011; Fucks y Schnack 2011). Los rodados en su mayoría son de basaltos y pórfidos, poseen tamaños que van entre los 2 y los $5 \mathrm{~cm}$ (con un diámetro medio de $3 \mathrm{~cm}$ ), y presentan un alto grado de redondeamiento y aplanamiento, característico de las gravas de playa (Angulo et al. 1978; Fidalgo y Porro 1981). La parte inferior es de color gris a gris blanquecino, y la superior, algo más limosa, de gris castaño a castaño (Fidalgo y Porro 1981).

Aflora en sectores de agradación marina en Bahía Rosas, Bahía Creek, Caleta de los Loros, Bajo de la Noria y en la bahía de San Antonio (Fidalgo y Porro 1981) (ver Figura
2). A pesar de que los rodados de esta formación habrían constituido una fuente de roca apta para la talla, su alto grado de redondeamiento y aplanamiento habría hecho difícil la talla de artefactos mediante la percusión directa. Presumiblemente, estos guijarros fueron aprovechados mediante la talla bipolar.

\section{Cordones litorales holocenos}

En la costa norte, entre Punta Villarino y Barranca Final, la playa alta presenta cordones de grava arenosa, que se extienden con continuidad. Los rodados y valvas de estos cordones provienen de la desagregación de la Fm. Baliza San Matías que aflora en toda la plataforma de abrasión de ola presente en este sector (Gelós et al. 1990). En las restantes playas, las psefitas sólo se presentan en escasa proporción, con excepción de Caleta de los Loros donde los rodados integran un nivel aterrazado por encima del nivel de playa actual (como se observa en el Bajo de la Quinta y en Bahía Rosas, que constituye una playa de gravas con marcada selección y gradación desde playa alta a baja). En estos tramos costeros, el origen de los rodados se vincula al retrabajo en la playa de los bloques derrumbados en sectores donde los rodados de la Fm. Tehuelche coronan el acantilado, redistribuyéndose posteriormente por deriva litoral (Gelós et al. 1990).

\section{Muestreos de materias primas}

A lo largo de toda la costa norte del golfo se han realizado un total de 22 muestreos de materias primas líticas en diferentes puntos del espacio (ver Figuras 3 y 4). Para estos muestreos se seleccionaron cordones litorales, depósitos de guijarros en playas actuales, en terrazas holocenas y un muestreo del Manto Tehuelche (Rodados Patagónicos) y de la formación Baliza San Matías. Todos estos sectores constituyen potenciales fuentes de materias primas líticas que pudieron haber sido aprovechadas en el pasado. Estos muestreos fueron realizados al azar (grillado del terreno y selección de puntos para la recolección de nódulos), por tiempo y por cantidad de nódulos con el objetivo de caracterizar las diferentes fuentes de rocas disponibles en el espacio (Figura 4).

En total se recuperaron 1285 nódulos de distintas materias primas (Tabla 1).

Los nódulos de mejor calidad (sensu Aragón y Franco 1997) que se encuentran en el área son los de calcedonia, sílex, xilópalo y jaspe, que son los que menos aparecen en las fuentes. Entre las vulcanitas la calidad varía de mala a muy buena, mientras que el resto de las rocas presenta calidades de buena a mala. En los muestreos se recuperaron principalmente nódulos de calidad mala $(n=511,39.77 \%)$ y regular $(n=309,24.05 \%)$ para la talla. Los nódulos de calidades buena $(n=237)$, muy buena $(n=152)$ y excelente $(n=76)$ para la talla aparecen en las fuentes en porcentajes menores (18.44\%, $11.83 \%$ y 


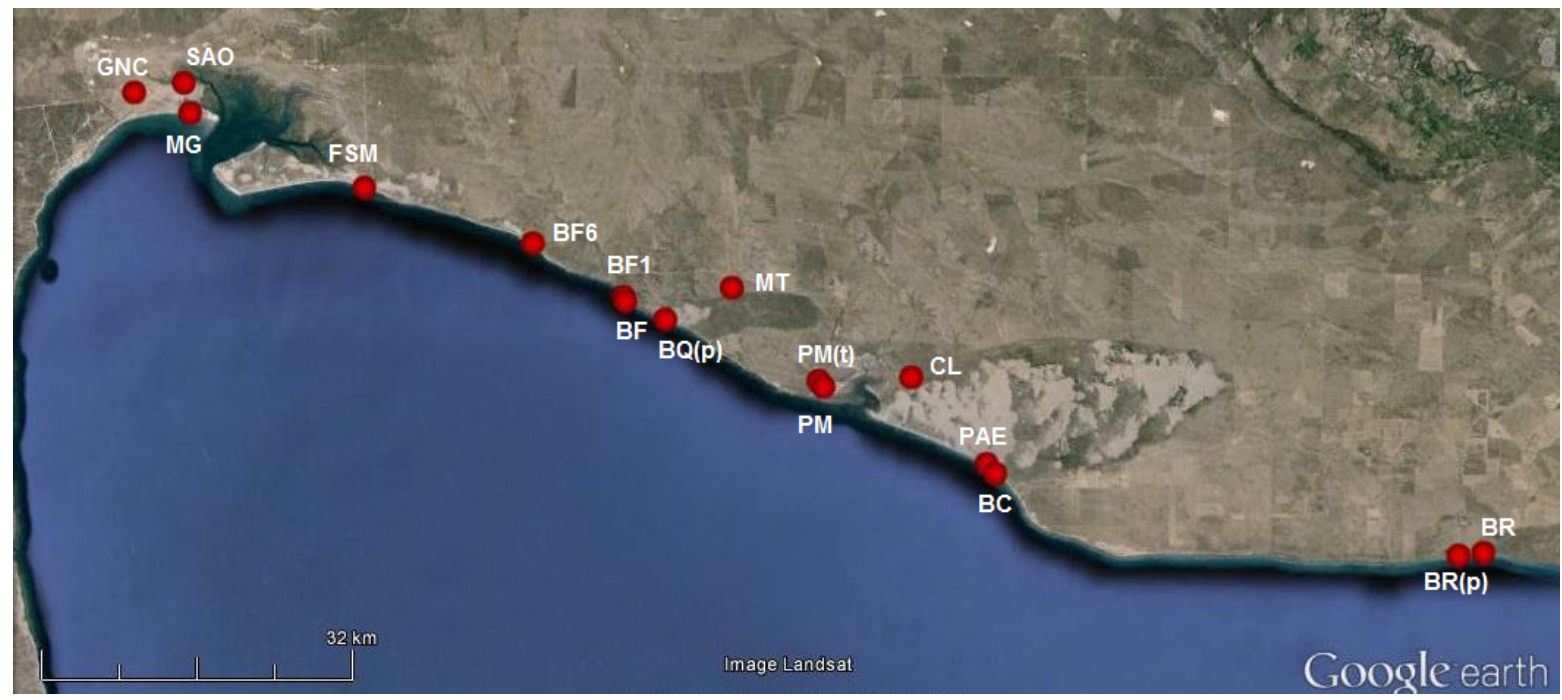

Figura 3. Muestreos de materias primas líticas. FSM: Faro San Matías (Fm. Baliza San Matías). BF1: Bahía Final 1. BF6: Bahía Final 6 (Sector 1). BQ(p): Bajo de la Quinta (paleoplaya). BR: Bahía Rosas. MG: Mar Grande (Sector 2). BF: Barranca Final. SAO: San Antonio Oeste (marisma norte). GNC: Sector Pesas GNC. MT: Manto Tehuelche (Rodados Patagónicos). BC: Bahía Creek (playa actual). CL: Caleta de los Loros (duna cercana a cordón fechado en $7300{ }^{14} \mathrm{C}$ AP). BR(p): Bahía Rosas (playa actual). PM: Punta Mejillón. PM(t): Punta Mejillón (terraza fechada en $5000{ }^{14} \mathrm{C}$ AP).

Figure 3. Lithic raw materials samples. FSM: Faro San Matías (Baliza San Matías fm). BF1: Bahía Final 1. BF6: Bahía Final 6 (Sector 1). BQ(p): Bajo de la Quinta (paleoplaya). BR: Bahía Rosas. MG: Mar Grande (Sector 2). BF: Barranca Final. SAO: San Antonio Oeste (marisma norte). GNC: Sector Pesas GNC. MT: Manto Tehuelche (Rodados Patagónicos). BC: Bahía Creek (current beach). CL: Caleta de los Loros (dune near to a ridge dated at $7300{ }^{14} \mathrm{C}$ AP). BR(p): Bahía Rosas (current beach). PM: Punta Mejillón. PM(t): Punta Mejillón (terrace dated at $5000{ }^{14} \mathrm{C} \mathrm{AP}$ ).

\section{$5.91 \%$, respectivamente).}

Las medidas totales de los nódulos fueron tomadas con calibre digital sobre las dimensiones máximas de cada pieza. El largo total cubre un rango va de 8 a $160 \mathrm{~mm}$, con un promedio de $60.54 \mathrm{~mm}$; el ancho de 10 a 107 $\mathrm{mm}$ con una media de $45.29 \mathrm{~mm}$; y los espesores se ubican entre 4 y $110 \mathrm{~mm}$ con una media de $27.32 \mathrm{~mm}$. El volumen promedio es de 99.9 cc. Estos resultados muestran que todas las medidas de estos nódulos tienen una dispersión de valores relativamente amplia, y que son nódulos de tamaño mediano y no muy espesos, lo cual facilitaría las tareas de talla. Sin embargo, estas rocas no habrían sido sencillas de tallar debido a la forma que presentan (ver Tabla 2).

La clasificación de la forma de los nódulos fue realizada según el criterio establecido por Zingg (1935) (en Shelley 1993). Esta autora determina las diferentes formas de los clastos a partir de dos valores, obtenidos de las relaciones axiales de las partículas (Figura 5). El primero deriva de la división del ancho por el largo de cada nódulo, y el segundo de la del espesor por el ancho. Estos valores son puestos luego dentro de un gráfico con dos ejes y de esta forma todas las partículas se pueden describir como pertenencientes a alguna de las siguientes categorías: globulares, discoidales, tabulares o proladas (Zingg 1935 en Shelley 1993) (Figura 5). Los términos "sub-globular" y "discoidal irregular" han sido introducido por nosotros para describir mejor la variabilidad en morfología de los rodados recuperados en los muestreos.
Tal como se desprende de la tabla, la morfología predominante de los nódulos es la prolada, la cual facilitaría la extracción de subproductos alargados aptos para la talla de instrumentos debido a que ya presentan plataformas naturales a partir de las cuales empezar la talla y formalizar nuevas plataformas de extracción (Shelley 1993). Sin embargo, las rocas que presentan esta forma tienen principalmente calidades malas y regulares para la talla (principalmente vulcanitas, $65 \%$ vs. 35\% de rocas de calidad buena, muy buena y excelente) con lo que no habrían sido las principales rocas usadas para la talla de instrumentos.

Las categorías siguientes son la forma globular y la subglobular (tomado y ampliado de Zingg 1935 en Shelley 1993) las cuales son difíciles de tallar debido a la dificultad para lograr plataformas de percusión adecuadas sobre las cuales golpear para extraer lascas o formas base que sirvan para la manufactura de instrumentos. Los nódulos de rocas que poseen esta forma son principalmente de calidad mala y regular (76\%). Las formas tabular, discoidal (Zingg 1935 en Shelley 1993) y discoidal irregular son las más idóneas para la talla de instrumentos ya que, al igual que las formas proladas, presentan plataformas naturales a partir de las cuales comenzar a extraer subproductos. Dentro de este grupo, 54\% son de calidad buena a excelente para la talla, mientras que el $46 \%$ restante son de calidad mala y regular. Dentro de este grupo es donde se encuentran en general las calcedonias, los jaspes y las rocas silíceas, que son las que presentan mejor calidad para la talla de entre el conjunto de nódulos recuperadas en los muestreos (ver Figura 6 para algunos ejemplos). 


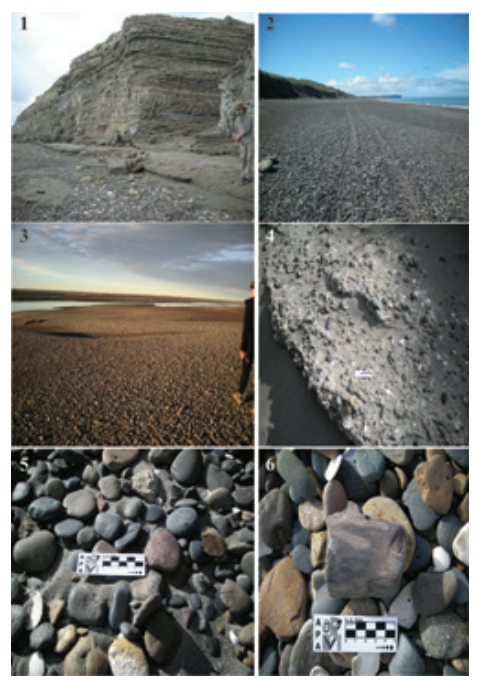

Figura 4. Algunas fuentes de materias primas líticas. 1. Bahía Creek (playa actual). 2. Bahía Rosas. 3. San Antonio Oeste (marisma). 4. Fm. Baliza San Matías (nótese la cementación de los clastos entre sí). 5. Nódulos en la paleoplaya de Bajo de la Quinta. 6. Nódulo de xilópalo en la playa frente a Bahía Final 1.

Figure 4. Some of the lithic raw material sources 1. Bahía Creek (current beach). 2. Bahía Rosas. 3. San Antonio Oeste (marisma). 4. Fm. Baliza San Matías (see the pebbles' cementation). 5. Pebbles at Bajo de la Quinta. 6. Xilopal pebble at the beach in Bahía Final 1.

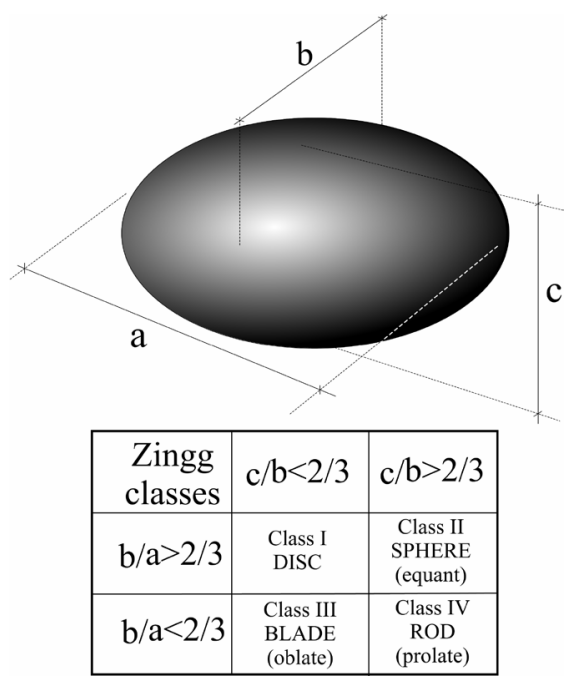

Figura 5. Ejes sobre los que se toman las medidas de los clastos para determinar su forma según Zingg (1935). DISC: discoidal. SPHERE: globular. BLADE: tabular. ROD: prolada.

Figure 5. Axis used to measure the pebbles in order to define their shape according to Zingg (1935). DISC: discoidal. SPHERE: globular. BLADE: tabular. ROD: prolate.

Respecto a las morfologías observadas en los muestreos de materias primas, el predominio de la forma prolada sobre las discoidales se registra en playas predominantemente gravosas. Allí existe un tamaño crítico para alcanzar la forma discoidal por tracción de olas: sólo algunos clastos la obtienen por deslizamiento de unos sobre otros, resultando más frecuentes las morfologías proladas (ver Bujalesky et al. 1994 y citas allí). En cambio, en playas de arena y gravas los clastos alcanzan morfologías más

\begin{tabular}{lcc}
\hline \multicolumn{1}{c}{ MATERIA PRIMA } & $\mathrm{n}$ & $\%$ \\
\hline Vulcanitas & 877 & 68,25 \\
Sílex o pedernal & 221 & 17,20 \\
Calcedonia & 80 & 6,23 \\
Cuarzo & 43 & 3,35 \\
Sedimentitas & 31 & 2,41 \\
Piroclastitas & 21 & 1,63 \\
Jaspe & 6 & 0,47 \\
Xilópalo & 3 & 0,23 \\
Plutonitas & 2 & 0,16 \\
M etamorfitas & 1 & 0,08 \\
TOTAL & 1285 & 100,00 \\
\hline
\end{tabular}

Tabla 1. Cantidad y porcentaje de nódulos recuperados en los muestreos de rocas realizados en la costa norte del golfo San Matías.

Table 1. Quantity and percentage of pebbles recovered in simples in the northern coast of San Matías Gulf.

\begin{tabular}{lcc}
\hline \multicolumn{1}{c}{ FORMA } & $\mathrm{n}$ & $\%$ \\
\hline Prolada & 519 & 40,39 \\
Globular & 409 & 31,82 \\
Sub-globular & 281 & 21,87 \\
Tabular & 131 & 10,19 \\
Discoidal & 83 & 6,46 \\
Discoidal irregular & 2 & 0,16 \\
TOTAL & 1285 & 100,00 \\
\hline
\end{tabular}

Table 2. Cantidad y porcentaje de nódulos discriminados por su forma.

Table 2. Quantity and percentage of pebbles according to their shape.

discoidales por la abrasión debida al deslizamiento. También hay que tener en cuenta en este caso la morfología original de los clastos derivada del acarreo glacifluvial/fluvial que generó los rodados de la Fm. Tehuelche, retrabajados luego por el mar, que heredaría predominantemente formas proladas y de elevada esfericidad.

Para la identificación petrográfica se han realizado un total de 23 cortes delgados que fueron identificados luego mediante microscopio petrográfico de luz polarizada en el Laboratorio de Petrografía del Departamento de Geología de la Facultad de Ciencias Exactas y Naturales de la Universidad de Buenos Aires. Las rocas para ser identificadas mediante esta metodología provienen de diferentes fuentes potenciales de materia prima lítica y, además, aparecen representadas en las localidades arqueológicas muestreadas en forma de diferentes artefactos líticos. Dentro de las identificaciones petrográficas, se determinaron diez vulcanitas (basaltos/ 

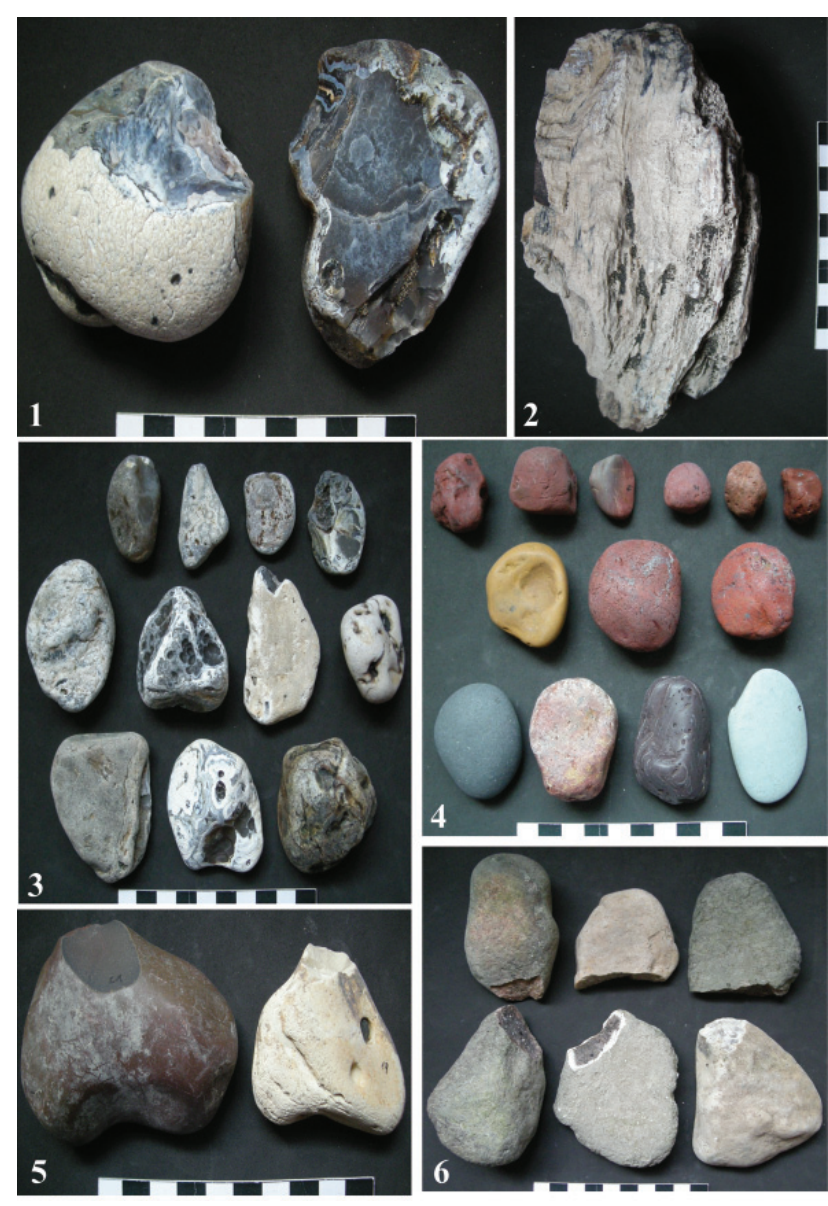

Figura 6. Algunos nódulos recuperados en los muestreos. 1 y 3. Calcedonias. 2. Xilópalo. 4. Jaspes, sílex y vulcanitas de grano fino. 5. Sílex. 6. Vulcanitas.

Figure 6. Some pebbles recovered at the samples. 1 and 3. Chalcedonies. 2. Xilopal. 4. Jasper, silex y DFGR (dark fine grained rocks). 5. Silex. 6 . Vulcanites.

andesitas y riolitas), ocho rocas sedimentarias (principalmente sílex y sílex biogénico), cuatro rocas piroclásticas (tobas y tobas silicificadas) y una metamórfica (cuarcita).

\section{La explotación de rocas en la costa norte del golfo San Matías}

A partir de muestreos realizados mediante cuadrículas de recolección llevadas a cabo en los sitios y transectas sistemáticas desarrolladas en el área de estudio de acuerdo con una metodología de muestreo de tipo distribucional (Franco y Borrerro 1999), se recuperaron un total de 5252 artefactos líticos (ver Tablas 3 y 4), que se analizaron siguiendo los criterios establecidos en Aschero (1975, rev. 1983). Los mismos han sido recuperados en loci pertenecientes a ambos bloques temporales de consumo de recursos (ver supra) y localizados tanto en contextos de dunas como de terrazas y paleoacantilados: dentro del bloque antiguo, loci de las localidades de San Antonio Oeste, Bajo de la Quinta, Bahía Final, Bahía Creek, Laguna de las Máquinas, Bahía Rosas y Caleta de los Loros, y del reciente de Faro San Matías, Saco Viejo, Bajo de la Quinta, Bahia Final, San Antonio Oeste y Bahía Creek. La muestra total se distribuyó en la forma presentada en la Tabla 3.

A pesar de que el tamaño total de las muestras es diferente entre bloques temporales (3660 artefactos en el bloque más temprano y 1719 para el más tardío), consideramos que los datos resultan suficientes para hacer estimaciones de tipo estadístico y para delinear tendencias en lo que hace al aprovisionamiento y explotación de rocas en la costa norte del golfo San Matías durante el Holoceno medio y tardío. Mientras que en el bloque tardío la roca más explotada fue el sílex, en el temprano fueron las vulcanitas. En segundo lugar lo ocupan las calcedonias en el bloque tardío y el sílex en el temprano. Finalmente, el tercer lugar es para las vulcanitas en el bloque tardío y para la calcedonia en el temprano. Esta afirmación permite, a priori, modificar las tendencias observadas previamente (ver Alberti 2012), de acuerdo con las cuales se había propuesto una explotación de rocas no locales en el bloque más temprano, como serían las calcedonias y la gran mayoría del sílex, y un giro hacia la explotación de rocas locales en tiempos más tardíos, una vez conocidas las fuentes de rocas del área (Alberti 2012). Para detectar diferencias estadísticamente significativas en el uso de rocas en uno y otro bloque temporal, se realizó un test de $\mathrm{Chi}^{2}$, comparando las frecuencias absolutas de vulcanitas, sílex y calcedonias. El resultado del test fue un valor de $\mathrm{X}^{2}$ de 306.59, con un $p$-valor $<0.01$. Esto quiere decir que las diferencias en las frecuencias que detectamos entre uno y otro bloque de consumo de los recursos marinos son estadísticamente significativas y que, efectivamente, estas rocas fueron usadas de forma diferencial en uno y otro momento de ocupación del área. Esto podría tener que ver con la circulación de los grupos dentro del ambiente y/o con un conocimiento cada vez mayor acerca de las rocas disponibles en la costa norte del golfo (Alberti 2012). Esta hipótesis está en evaluación.

En relación con los cambios detectados en el uso de rocas, es necesario mencionar las transformaciones que se sucedieron en la línea de costa a lo largo del Holoceno. En este sentido, Favier Dubois (2013) sostiene que con el primer pulso de descenso y estabilidad del nivel del mar al que refieren los trabajos geológicos en Patagonia (i.e. Codignotto et al. 1990; Monti 2000; Schellmann y Radtke 2010), entre 6.500/6.000 y 3.800/3.200 ${ }^{14} \mathrm{C} \mathrm{AP}$, pudo haberse iniciado el relleno de los entrantes marinos en la costa norte del golfo. Con el segundo pulso de descenso, luego del $3.200{ }^{14} \mathrm{C} \mathrm{AP}$, habría comenzado la acreción costera en una escala más amplia, dando lugar a la formación de cordones litorales en costas abiertas los cuales habrían funcionado como proveedores de rocas para los momentos más tardíos de la ocupación litoral (Favier Dubois 2013; Favier Dubois y Kokot 2011; Favier Dubois y Scartascini 2012). En el caso del área de Bahía Final 6, por ejemplo, estos cordones habrían alcanzado 
una extensión de $600 \mathrm{~m}$ de ancho (Alberti 2012). De esta manera, para los momentos tardíos habría existido una disponibilidad de rocas mayor que para momentos previos, las cuales habrían sido de más fácil extracción y aprovechamiento. Cabe destacar que las rocas que

\begin{tabular}{lcccc}
\hline \multirow{2}{*}{ M ATERIA PRIM A } & \multicolumn{2}{c}{ Bloque antiguo } & \multicolumn{2}{c}{ Bloque tardío } \\
& $\mathrm{n}$ & $\%$ & $\mathrm{n}$ & $\%$ \\
\hline Vulcanitas & 1582 & 43,22 & 324 & 18,85 \\
Sílex o pedernal & 1401 & 38,28 & 921 & 53,58 \\
Calcedonia & 558 & 15,25 & 407 & 23,68 \\
Jaspe & 38 & 1,04 & 2 & 0,12 \\
Sedimentitas & 35 & 0,96 & 34 & 1,98 \\
Piroclastitas & 13 & 0,36 & 3 & 0,17 \\
Cuarzo & 10 & 0,27 & 1 & 0,06 \\
Obsidiana negra & 8 & 0,22 & 3 & 0,17 \\
No diferenciadas & 6 & 0,16 & 6 & 0,35 \\
Ópalo & 6 & 0,16 & 5 & 0,29 \\
Plutonitas & 2 & 0,05 & 3 & 0,17 \\
Xilópalo & 1 & 0,03 & 5 & 0,29 \\
Cuarcita & 0 & 0,00 & 5 & 0,29 \\
TOTAL & 3660 & 100 & 1719 & 100 \\
\hline
\end{tabular}

Tabla 3. Frecuencia y porcentaje de artefactos provenientes de los sitios de la costa norte del golfo San Matías, divididos por tipo de materia prima.

Table 3. Frequency and percetage of artifacts recovered at the archaeological sites of the northern coast of San Matías Gula, diveded according to lithic raw materials.

\begin{tabular}{lcccccc}
\hline \multirow{2}{*}{ M ATERIA PRIM A } & \multicolumn{2}{c}{ DESECHOS } & \multicolumn{2}{c}{ INSTRUM ENTOS } & \multicolumn{2}{c}{ NUCLEOS } \\
& $\mathrm{n}$ & $\%$ & $\mathrm{n}$ & $\%$ & $\mathrm{n}$ & $\%$ \\
\hline Sílex o pedernal & 1975 & 43,82 & 111 & 42,05 & 197 & 40,96 \\
Vulcanitas & 1543 & 34,24 & 91 & 34,47 & 199 & 41,37 \\
Calcedonia & 850 & 18,86 & 42 & 15,91 & 65 & 13,51 \\
Sedimentitas & 49 & 1,09 & 12 & 4,55 & 4 & 0,83 \\
Jaspe & 29 & 0,64 & 1 & 0,38 & 9 & 1,87 \\
Piroclastitas & 12 & 0,27 & 1 & 0,38 & 1 & 0,21 \\
Obsidiana negra & 11 & 0,24 & 0 & 0,00 & 0 & 0,00 \\
No diferenciadas & 11 & 0,24 & 0 & 0,00 & 1 & 0,21 \\
Cuarzo & 10 & 0,22 & 0 & 0,00 & 1 & 0,21 \\
Ópalo & 9 & 0,20 & 0 & 0,00 & 2 & 0,42 \\
Xilópalo & 6 & 0,13 & 0 & 0,00 & 0 & 0,00 \\
Plutonitas & 2 & 0,04 & 1 & 0,38 & 2 & 0,42 \\
Cuarcita & 0 & 0,00 & 5 & 1,89 & 0 & 0,00 \\
TOTAL & 4507 & 100 & 264 & 100 & 481 & 100 \\
\% DEL TOTAL & 85,81 & & 5,02 & & 9,15 & \\
\hline
\end{tabular}

conforman estos cordones son predominantemente volcánicas y de calidad regular a buena para la talla.

Respecto de las categorías artefactuales, las materias primas se distribuyeron como se presenta en la Tabla 4

Tal como se desprende de la tabla 4, el porcentaje mayor corresponde a los desechos, que representan el $86 \%$ de la muestra, seguidos de los núcleos (9\%) y en último lugar los instrumentos (5\%). El mayor porcentaje de desechos corresponde al sílex, seguido de las vulcanitas y en tercer lugar las calcedonias, independientemente del bloque considerado. En los instrumentos el orden es el mismo, pero en los núcleos, aunque con una mínima diferencia, el primer lugar lo ocupan las vulcanitas, quedando relegado el sílex a la segunda posición. El resto de las rocas se encuentra representado con porcentajes muy bajos de aparición. Así, a partir de los análisis artefactuales, se puede afirmar que en la costa norte del golfo San Matías, independientemente del momento considerado, fueron las rocas silíceas las más utilizadas (ver Tabla 4), que aparecen en las fuentes en porcentajes muy bajos, seguidas de las vulcanitas, que serían principalmente de origen local y de menor calidad para la talla que las primeras, y finalmente las calcedonias, de baja frecuencia en las fuentes locales (distantes menos de $5 \mathrm{~km}$ desde los sitios) y de tamaños, en general, pequeños (ver más arriba).

\section{Conclusiones}

La costa norte del golfo San Matías presenta abundantes fuentes de materias primas líticas de tipo principalmente secundarias, con rocas de diferentes orígenes (volcánicas, sedimentarias, piroclásticas y metamórficas). Esto implica que el aprovisionamiento de rocas no habría constituido un factor de riesgo para los grupos cazadores-recolectores que habitaron el área durante el Holoceno medio y tardío (Alberti 2012; Cardillo 2013; Favier Dubois et al. 2009b). A partir de la consulta de la información geológica disponible (ver supra) y de la realización de muestreos de estas fuentes en diferentes puntos del espacio, se ha podido caracterizar la amplia variedad de rocas, de diferentes calidades para la talla, que estuvieron disponibles para los grupos que habitaron el área. En este sentido, en la base regional de recursos líticos del área (sensu Ericson 1984), las rocas predominantes son las

Tabla 4. Categorías de artefactos divididos por materia prima.

Table 4. Artifacts classified according to lithic raw materials. 
vulcanitas ácidas y básicas. También hay disponibles rocas sedimentarias tanto químicas como clásticas, de calidad relativamente buena para la talla que, sin embargo, no fueron intensamente aprovechadas. Otras rocas, como las calcedonias y el sílex, formarían parte de esta base regional de rocas pero en proporciones mucho menores. En particular es de destacar que su aparición en los sitios es mayor a la esperada teniendo en cuenta el análisis de las fuentes de materias primas, con lo que podría ser que estas rocas hubiesen sido traídas a la zona desde regiones aledañas (ver más adelante).

Debido a que todas las fuentes de materias primas relevadas son secundarias, no hemos podido comprobar la efectiva explotación por parte de los grupos que habitaron la costa. Tal como se ha expresado más arriba, la explotación de las rocas varió a lo largo del tiempo, pese a que en la base regional no se habrían registrado grandes cambios (a excepción de la formación de los cordones litorales los que, sin embargo, no habrían variado la oferta de tipos de rocas disponibles, aunque sí la cantidad y disponibilidad inmediata). En este sentido, podemos considerar que la base regional de recursos líticos de la costa norte del golfo San Matías se mantuvo relativamente constante a lo largo del tiempo que duró la ocupación en el área.

Independientemente de la calidad de los nódulos y de su amplia disponibilidad, la forma y el tamaño de los mismos habría condicionado su selección y explotación posterior. A diferencia del tamaño, que habría facilitado las tareas de talla y el transporte de los nódulos hacia otros puntos del espacio, la forma no habría sido la adecuada para estas actividades debido a que las formas proladas y globulares son las más difíciles de tallar (ver supra). De acuerdo con su calidad y forma, las rocas más aptas para la talla habrían sido las calcedonias, los jaspes y el sílex, que no son las más abundantes en las fuentes de materias primas de la costa norte del golfo. Es importante mencionar que las morfologías de los clastos aparecen en las playas en distribuciones diferenciales, de acuerdo con las características de las mismas, con lo que no en todos los puntos del espacio las formas obtenidas habrían sido las mismas. Este es un factor más que se combina con el tamaño de las rocas, el tipo de materia prima y su calidad para la talla.

A pesar de la abundancia general de rocas en la costa del golfo, la selección para la talla es evidente: principalmente rocas locales en el bloque más temprano y no locales en el tardío. Aunque las calcedonias y el sílex aparecen en las fuentes de la costa norte, son rocas cuyo origen es considerado no local debido a que su aparición en los sitios es mucho mayor a la registrada en las fuentes. Estas rocas podrían provenir de la costa oeste donde aparecen con mayor frecuencia (Alberti y Cardillo 2014), o quizás de lugares más distantes. Por ejemplo, se ha relevado una fuente de calcedonia en la zona de la meseta de Somuncurá (Miotti et al. 2004), distante ca. 200 km lineales del área de estudio. La evaluación de la posible procedencia de las calcedonias está aún en proceso. Es necesario tener en cuenta que en el bloque más tardío la disponibilidad de materias primas líticas fue mayor debido a los procesos de acreción costera, como ya se ha explicitado, pero esto no modificó los tipos de rocas disponibles. Esto se hace evidente, particularmente, al considerar los porcentajes y frecuencias de aparición de los distintos tipos de rocas en el conjunto general de artefactos.

En el presente trabajo hemos delineado las tendencias generales del aprovechamiento de rocas en la costa norte del golfo San Matías. Esta información debe ser complementada con el estudio de las fuentes de materias primas líticas aptas para la talla disponibles en el sector oeste de dicho golfo (Alberti y Cardillo 2014). La continuación de los trabajos en el mencionado sector permitirá obtener un panorama más claro y amplio acerca del aprovechamiento de rocas por parte de los grupos cazadores-recolectores que habitaron la costa rionegrina del golfo San Matías durante el Holoceno medio y tardío.

\section{Agradecimientos}

A los miembros del equipo de investigación por la colaboración en las tareas de campo. Al Dr. Marcelo Cardillo por la ayuda brindada para la realización de los análisis estadísticos. A los Dres. Vanesa Litvak y Oscar Limarino por las correcciones y comentarios hechos sobre las identificaciones de las rocas en cortes delgados. A los proyectos PIP CONICET 6415, PICT ANPCyT 38264 y PIP CONICET 112-200801-00756 que permitieron financiar estas investigaciones. A los evaluadores anónimos cuyos comentarios y sugerencias ayudaron a mejorar sensiblemente este trabajo.

Olavarría, 29 de octubre 2013

\section{Bibliografía}

Alberti, J. 2012. Fuentes de rocas y uso de materias primas líticas en Bahía Final 6, costa norte del golfo San Matías (Río Negro, Argentina). Intersecciones en Antropología, 13:237-249.

Alberti, J. y M. Cardillo. 2014. Primary and secondary lithic raw material sources along the western coast of San Matías Gulf (Río Negro province, Argentina): a first approach to their spatial variability. MS.

Andreis, R.1965. Petrografía y paleocorrientes de la Formación Río Negro, tramo Gral. Conesa - boca del Río Negro. Revista del Museo de La Plata, 5 (Geología)36: 20-73.

Angulo, R., F. Fidalgo, M. Gomez Peral y E. Schnack. 1978. Las ingresiones marinas cuaternarias en la bahía de San Antonio y sus vecindades, provincia de Río Negro. En Actas del VII Congreso Geológico Argentino, Vol. 1:271-283. Neuquén.

Angulo, R., F. Fidalgo, M. Gomez Peral y E. Schnack. 1981. Geología y geomorfología del bajo de San Antonio y 
alrededores, provincia de Río Negro. Estudios y Documentos 8. Viedma, Centro de Investigaciones Científicas - Secretaria de planeamiento

Aragón, E. y N. Franco. 1997. Características de rocas para la talla por percusión y propiedades petrográficas. Anales del Instituto de la Patagonia, 25: 187-189.

Aschero, C. 1975. Ensayo para una clasificación morfológica de artefactos líticos aplicada a estudios tipológicos comparativos. Informe presentado al CONICET. MS.

1983. Ensayo para una clasificación morfológica de artefactos líticos. Apéndice $A$ y $B$. Cátedra de Ergología y Tecnología. Facultad de Filosofía y Letras, Universidad de Buenos Aires. Informe presentado al CONICET. MS.

Bujalesky, G., A. Coronato, C. Roig y J. Rabassa. 1994. Forma de rodados de ambientes glacigénicos del Pleistoceno y costeros actuales del lago Fagnano, Tierra del Fuego. Revista de la Asociación Geológica Argentina, 49 (1-2): 154-164.

Busteros, A., R. Giacosa y H. Lema. 1998. Hoja Geológica 4166-IV, Sierra Grande, provincia de Río Negro. Buenos Aires, Boletín 241. Instituto de Geología y Recursos Minerales, Servicio Geológico Minero Argentino. 75p

Camacho, H. 1967. Las transgresiones del Cretácico Superior y Terciario de la Argentina. Revista de la Asociación Geológica Argentina, 22(4):253-280.

Cardillo, M. 2013. Cambios en el paisaje, uso del espacio y conjuntos líticos promediados en la costa norte del golfo San Matías (Río Negro, Argentina) durante el Holoceno medio-tardío. Comechingonia Virtual, 1:1-26.

Codignotto, J., C. Beros y L. Trebino 1987. Nuevo método cronoestratigráfico, morfocronología en secuencia deposicional cordoniforme. Revista de la Asociación Geológica Argentina, 42(3-4):462-468.

Cortelezzi, C., O. De Salvo y F. De Francesco. 1963. Estudio de las gravas tehuelches de la región comprendida entre el río Colorado y el río Negro, desde la costa de la provincia de Buenos Aires, hasta Choele Choele. En Actas de las Segundas Jornadas Geológicas Argentinas, Tomo II, pp. 65-87, Salta.

Del Río, J., M. Bó, M. López de Armentía, J. Álvarez, J. Martínez Arca, C. Wagner y M. Camino. 2005. Geomorfología descriptiva y ambiental de la costa oriental del golfo San Matías y la desembocadura del río Negro. R. Masera, J. Lew y G. Serra Peirano (eds.), Las mesetas patagónicas que caen al mar: la costa rionegrina, 201-220, Gobierno de Río Negro, Viedma.

Ericson, J. 1984. Toward the analysis of lithic reduction systems. J. Ericson y B. Purdy (eds.), Prehistoric quarries and lithic production, 1-19. Cambridge University Press, Cambridge.

Etcheverría, M., A. Folguera, C. Dal Molín, M. Dalponte y G. Ferro. 2006. Hojas Geológicas 4163-II/IV y I/III, Viedma y General Conesa. Provincias de Río Negro y Buenos Aires. Boletín 366, Instituto de Geología y Recursos Minerales, Servicio Geológico Minero Argentino, Buenos Aires.

Favier Dubois, C. 2013. Hacia una cronología del uso del espacio en la costa norte del golfo San Matías (Río Negro, Argentina): sesgos geológicos e indicadores temporales. F. Zangrando, R. Barberena, A. Gil, G. Neme, M. Giardina, L. Luna, C. Otaola, S. Paulides, L. Salgán y A. Tivoli (eds.), Tendencias teórico-metodológicas y casos de estudio en la arqueología de Patagonia, 87-96, Museo de Historia Natural de San Rafael y Sociedad Argentina de Antropología, San Rafael.

Favier Dubois, C., F. Borella y R. Tykot. 2009a. Explorando tendencias en el uso humano del espacio y los recursos en el litoral rionegriono (Argentina) durante el Holoceno medio y tardío. M. Salemme, F. Santiago, M. Álvarez, E. Piana, M. Vázquez y E. Mansur (eds.), Arqueología de la Patagonia - Una mirada desde el último confín, 985-997, Editorial Utopías, Ushuaia.

Favier Dubois, C., F. Borella, L. Manzi, M. Cardillo, S. Lanzellotti, F. Scartascini, M. Carolina y E. Borges Vaz. 2008. Aproximación regional al registro arqueológico de la costa rionegrina. I. Cruz y S. Caracotche (eds.), Arqueología de la Costa Patagónica. Perspectivas para la conservación, 50-68. Universidad Nacional de la Patagonia Austral, Río Gallegos.

Favier Doubois, C. y R. Kokot. 2011. Changing scenarios in Bajo de la Quinta (San Matías Gulf, Northern Patagonia, Argentina): impact of geomorphologic processes in subsistence and human use of coastal habitats. Quaternary International, 245:103-110.

Favier Dubois, C. y F. Scartascini. 2012. Intensive fishery scenarios on the North Patagonian coast (Río Negro, Argentina) during the Mid-Holocene. Quaternary International, 256:62-70.

Favier Dubois, C., C. Stern y M. Cardillo. 2009b. Primera caracterización de los tipos de obsidiana presentes en la costa rionegrina. M. Salemme, F. Santiago, M. Álvarez, E. Piana, M. Vázquez y E. Mansur (eds.), Arqueología de la Patagonia - Una mirada desde el último confín, 349-359, Editorial Utopías, Ushuaia.

Feruglio, E. 1950. Descripción Geológica de la Patagonia. Ministerio de industria y comercio - Dirección General de Yacimientos Petrolíferos Fiscales, Buenos Aires.

Fidalgo, F. y N. Porro. 1981. Descripción geológica de la hoja 39j, San Antonio Oeste, provincia de Río Negro. Buenos Aires. Trabajo inédito.

Fidalgo, F. y J. Rabassa. 1984. Geología y recursos naturales de la provincia de Río Negro. Los depósitos cuaternarios. IX Congreso Geológico Argentino, Relatorio I:291-316. San Carlos de Bariloche.

Fidalgo, F. y J. C. Riggi. 1970. Consideraciones geomórficas y sedimentológicas sobre los rodados patagónicos. Revista de la 
Asociación Geológica Argentina, 25(4):430-443.

Franchi, M. 1983. Descripción geológica de las Hojas 39m y 40m, Viedma, y 39n, isla San Blás. Provincias de Buenos Aires y Río Negro. Servicio Geológico Nacional, Buenos Aires. Trabajo inédito.

Fucks, E. y E. Schnack. 2011. Evolución geológica y geomorfológica del sector norte del golfo San Matías. Actas del XVIII Congreso Geológico Argentino, 273-274, Neuquén.

Franco, N. y L. Borrero. 1999. Metodología de análisis de la estructura regional de recursos líticos. C. Aschero, A. Korstanje y P. Vuoto (eds.), En los Tres Reinos. Prácticas de Recolección en el Cono Sur de América, 27-37. Magna Publicaciones, San Miguel de Tucumán.

Gelós, E., J. Spagnuolo y R. Schillizzi. 1990. Caracteres texturales y mineralógicos de sedimentos de playa de la costa norte del golfo San Matías. Revista de la Asociación Argentina de Mineralogía, Petrología y Sedimentología, 21(1/4):41-52.

Gelós, E., R. Schillizzi y J. Spagnuolo. 1992. El Mesozoico superior-Cenozoico de la costa occidental del Golfo San Matías. Revista de la Asociación Geológica Argentina, 47(4):423-426.

González Díaz, E. y E. Malagnino. 1984. Geomorfología de la provincia de Río Negro. Actas del IX Congreso Geológico Argentino. San Carlos de Bariloche.

Kaasschieter, J. 1965. Geología de la Cuenca del Colorado. Actas de las Segundas Jornadas Geológicas Argentinas, Vol. III:251-269, Buenos Aires.

Malumián, N. y C. Nañez. 2011. The Late Cretaceous-Cenozoic transgressions in Patagonia and the Fuegian Andes: foraminifera, paleoecology and paleogeography. Biological Journal of the Linnean Society, 103:269-288.

Marani, H. y F. Scartascini. 2011. Integrando datos zooarqueológicos e isotópicos para una discusión de la subsistencia en la costa norte del Golfo San Matías (Río Negro). Trabajo presentado en el II Congreso Nacional de Zooarqueología Argentina. Olavaria, Facultad de Ciencias Sociales, Universidad de Centro de la Provincia de Buenos Aires. 10-14/05/2011.

Martínez, H., C. Náñez, A. Lizuain, C. Dal Molín y A. Turel. 2001. Hoja Geológica 4166-II, San Antonio Oeste. Provincia de Río Negro. Boletín 254, Instituto de Geología y Recursos Minerales, Servicio Geológico Minero Argentino, Buenos Aires.

Miotti L., M. Salemme, D. Hermo, L. Magnin y J. Rabassa. 2004. Yamnago 137 años después: otro lenguaje para la misma región. T. Civalero, P. Fernández y G. Guráieb (eds.), Contra Viento y Marea. Arqueología de Patagonia, 775-796, Instituto Nacional de Antropología y Sociedad Argentina de Antropología, Bs As.

Monti, A. J. 2000. Edades 14C y ciclicidad de la acreción en depósitos costeros elevados, Bahía Engaño, Chubut. Revista de la Asociación Geológica Argentina, 55(4): 403-406.

Rostami, K., W. Peltier y A. Manzini. 2000. Quaternary marine terraces, sea-level changes and uplift history of Patagonia, Argentina: comparisons with predictions of the ICE-4G (VM2) model of the global process of glacial isostatic adjustment. Quaternary Science Reviews, 19:1495-1525.

Schellmann, G., y U. Radtke. 2010. Timing and magnitude of Holocene sea-level changes along the middle and south Patagonian Atlantic coast derived from beach ridge systems, litoral terraces and valley-mouth terraces. Earth-ScienceReviews, 103:1-30.

Orlando, M. 2009. Instrumentos de molienda y uso del espacio en la costa norte de la provincia de Río Negro: una primera aproximación. M. Salemme, F. Santiago, M. Álvarez, E. Piana, M. Vázquez y E. Mansur (eds.), Arqueología de la Patagonia - Una mirada desde el último confín, 1127-1140, Editorial Utopías, Ushuaia.

Sanchez, L. 1973. Geología litoral. Relevamiento ecológico y tipificación de las comunidades del litoral marítimo de la provincia de Río Negro, con especial referencia al establecimiento de áreas de cultivo para especies de interés comercial, 112-141, Instituto de Biología Marina. Asesoría de desarrollo de Río Negro - Consejo Federal de Inversiones, Buenos Aires.

Scartascini, F. 2010. "Explotación de peces en la costa norte del golfo San Matías (Río Negro): cambios y continuidades en la subsistencia y uso del espacio costero". Facultad de Filosofía y Letras, Universidad de Buenos Aires, Argentina, 129 páginas. Tesis de Licenciatura. Facultad de Filosofía y Letras, Universidad de Buenos Aires.

Schellmann, G. y T. Radtke. 2010. Timing and magnitude of Holocene sea-level changes along the middle and south Patagonian Atlantic coast derived from beach ridge systems, littoral terraces and valley-mouth terraces. Earth-Science Reviews, 103:1-30.

Shelley, P. 1993. A Geoarchaeological Approach to the Analysis of Secondary Lithic Deposits. Geoarchaeology: An International Journal, 8(1):59-72.

Weber, E. 1983. Descripción geológica de la Hoja 40j, Cerro El Fuerte, provincia de Río Negro. Boletín 196. Ministerio de Economía, Secretaría de Estado de Industria y Minería, Subsecretaría de Minería, Servicio Geológico Nacional, Bs. As.

Wichman, R. 1918. Estudios geológicos e hidrológicos en la región comprendida entre boca del Río Negro, San Antonio y Choele-Choel. Anales del Ministerio de Agricultura de la Nación - Sección Geología, Mineralogía y Minería, 13(3):1-49.

Zavala, C. y H. Freije. 2005. Geología de los acantilados rionegrinos. R. Masera, J. Lew y G. Serra Peirano (eds.), Las mesetas patagónicas que caen al mar: la costa rionegrina, 185-200, Gobierno de Río Negro, Viedma. 